\title{
Video Article \\ Simultaneous Transcranial Alternating Current Stimulation and Functional Magnetic Resonance Imaging
}

\author{
Kathleen A. Williams ${ }^{1}$, Yuranny Cabral-Calderin ${ }^{1,3}$, Carsten Schmidt-Samoa ${ }^{1}$, Christiane Anne Weinrich ${ }^{1,2}$, Peter Dechent ${ }^{1}$, Melanie Wilke ${ }^{1,3,4}$ \\ ${ }^{1}$ Department of Cognitive Neurology, University Medicine Goettingen \\ ${ }^{2}$ Department of Neurology, University Medicine Goettingen \\ ${ }^{3}$ German Primate Center, Leibniz Institute for Primate Research \\ ${ }^{4}$ DFG Center for Nanoscale Microscopy \& Molecular Physiology of the Brain (CNMPB)
}

Correspondence to: Kathleen A. Williams at katie.aw@gmail.com

URL: https://www.jove.com/video/55866

DOI: doi:10.3791/55866

Keywords: Behavior, Issue 124, transcranial alternating current stimulation (tACS), functional magnetic resonance imaging (fMRI), oscillation, frequency, noninvasive brain stimulation

Date Published: 6/5/2017

Citation: Williams, K.A., Cabral-Calderin, Y., Schmidt-Samoa, C., Weinrich, C.A., Dechent, P., Wilke, M. Simultaneous Transcranial Alternating Current Stimulation and Functional Magnetic Resonance Imaging. J. Vis. Exp. (124), e55866, doi:10.3791/55866 (2017).

\section{Abstract}

Transcranial alternating current stimulation (tACS) is a promising tool for noninvasive investigation of brain oscillations. TACS employs frequency-specific stimulation of the human brain through current applied to the scalp with surface electrodes. Most current knowledge of the technique is based on behavioral studies; thus, combining the method with brain imaging holds potential to better understand the mechanisms of tACS. Because of electrical and susceptibility artifacts, combining tACS with brain imaging can be challenging, however, one brain imaging technique that is well suited to be applied simultaneously with tACS is functional magnetic resonance imaging (fMRI). In our lab, we have successfully combined tACS with simultaneous fMRI measurements to show that tACS effects are state, current, and frequency dependent, and that modulation of brain activity is not limited to the area directly below the electrodes. This article describes a safe and reliable setup for applying tACS simultaneously with visual task fMRI studies, which can lend to understanding oscillatory brain function as well as the effects of tACS on the brain.

\section{Video Link}

The video component of this article can be found at https://www.jove.com/video/55866/

\section{Introduction}

Transcranial alternating current stimulation (tACS) is a noninvasive brain stimulation technique with promise for investigating neural oscillations and frequency-specific brain functions in healthy individuals as well as to study and modulate oscillations in clinical populations ${ }^{1}$. Using two or more conductive electrodes placed on the scalp, low current (1-2 mA peak-to-peak) sinusoidal waves are applied to the brain at a desired frequency to interact with ongoing neural oscillations. TACS studies have measured frequency- and task-specific behavioral or cognitive modulations including but not limited to motor function ${ }^{2}$, working memory performance ${ }^{3}$, somatosensation ${ }^{4}$, and visual perception ${ }^{5,6,7}$. Applying alternating current in a noninvasive manner has also resulted in functional improvement in neurological patients, such as tremor reduction in Parkinson's disease ${ }^{8}$, improved vision in optical neuropathy ${ }^{9}$, and improved rate of speech, sensory, and motor recovery after stroke ${ }^{10}$. Despite an increasing number of studies using tACS for research and evidence of its therapeutic potential in clinical settings, the effects of this technique are not fully characterized, and its mechanisms are not completely understood.

Simulations and animal studies can provide insight into the effects of alternating current stimulation at the cellular or neural network level under controlled conditions ${ }^{11,12}$, but given the state-dependence of effective stimulation techniques ${ }^{13,14}$, such studies do not reveal the whole picture. Combining tACS with neuroimaging techniques like electroencephalography (EEG) ${ }^{15,16,17}$, magnetoencephalography (MEG) ${ }^{18,19,20}$, or functional magnetic resonance imaging (fMRI) ${ }^{21,22,23,24}$ can inform about systems-level modulation of brain function. However, each combination comes with technological challenges, mainly due to stimulation-induced artifacts in the measurement of frequencies of interest ${ }^{15}$. Although the temporal resolution of fMRI cannot match EEG or MEG measurements, its spatial coverage and resolution in cortical and subcortical brain regions is superior.

Recently, in a combined tACS-fMRI study, we showed that effects of tACS on the blood oxygenation level dependent (BOLD) signal measured with fMRI are both frequency and task specific, and that the stimulation does not necessarily exert its greatest effect directly underneath the electrodes, but in regions more distant from the electrodes ${ }^{22}$. In a following study, we investigated the effect of tACS electrode position and frequency on network function using amplitude of low frequency fluctuations and resting-state functional connectivity, including using correlation seeds of the most directly stimulated regions, as derived from subject-based current density simulations. Most notably in this study, alpha $(10 \mathrm{~Hz})$ and gamma $(40 \mathrm{~Hz})$ stimulation often elicited opposite effects in network connectivity or on regional modulation ${ }^{23}$. Additionally, the resting-state network that was most affected was the left fronto-parietal control network. These studies highlight the potential for using fMRI 
to determine optimal parameters for effective, controlled stimulation. Also, they contribute to evidence that, aside from controlled parameters, such as task condition and timing, stimulation frequency, and electrode positions, there are subject-specific factors that influence the success of tACS. Examples of subject characteristics that translate as uncontrollable variables in optimizing stimulation parameters are intrinsic functional connectivity, endogenous oscillation peak frequency (e.g., individual alpha frequency), and skull and skin thickness ${ }^{25}$. Considering the current body of literature pertaining to tACS, more studies combining tACS with neural measurements such as neuroimaging are required to establish comprehensive procedures for effective brain stimulation techniques.

Herein, we describe a safe and reliable setup for experiments applying tACS simultaneously with fMRI of a visual task, with focus on aspects of the setup and execution that yield successfully synchronized tACS with artifact-free acquisition of fMRI data.

\section{Protocol}

Conduct all experiments in accordance with institutional ethics committee guidelines. For all studies mentioned in this manuscript, all procedures were performed according to the declaration of Helsinki and approved by the local Ethics Committee of the University Medical Center Göttingen.

\section{Stimulation and Computer Setup Prior to Experiment}

\section{Stimulator setup}

NOTE: The stimulator used for this fMRI experiment is a specially designed magnetic resonance (MR)-compatible system equipped with an MR-safe inner filter box, an outer filter box, safety resistors, coupled cables, and MR-safe materials. Some instructions pertain specifically to the manufacturer's directions, and these may vary when using another stimulator, so take care to follow equipment instructions provided by the manufacturer that may constitute exceptions to this setup. Figure 1A shows the stimulator components used in this experimental setup.

1. Navigate through the menu of the stimulator to program the desired experimental parameters (refer to user's manual for details). For example, for a stimulation frequency of $10 \mathrm{~Hz}$, program 10 cycles for ramp-up/down time of $1 \mathrm{~s}, 300$ sinusoidal cycles for $30 \mathrm{~s}$ of stimulation, current strength equal to $1,000 \mu \mathrm{A}$, and repetitive trigger mode, as conducted for our experiments unless otherwise noted Save the program to load for each time the experiment is run thereafter.

2. Connect the stimulus presentation computer trigger output to the stimulator using a BNC cable.

3. Place a nonmagnetic, shielded local area network (LAN) cable through the radio frequency (RF) waveguide tube from the inside of the scanner room. To avoid resonant capacitive coupling, ensure that the cable is free from loops and placed along the wall of the room, leading to the rear of the magnet bore and along the right-side scanner bed railing inside the bore, leading to the position of the inner filter box (see Figure 1C and safety note in Step 2.4 regarding cable position). Secure the cable with tape placed intermittently along the length of it.

2. Load the visual stimulus program on a designated presentation computer that is separate from the scanner control computer. As depicted in Figure 1C, connect the presentation computer to the scanner trigger output via an optical-to-electrical converter and to an output device (i.e., projector) that is placed in a shielded case or outside the magnet room. Use nonmagnetic mirrors to direct the projection onto a screen inside the scanner bore.

\section{Subject Arrival and Preparation}

1. Pre-screen recruited subjects for any contraindications for MR scanning (e.g., no metal implants, no claustrophobia, experiment-specific subject prerequisites) as well as for tACS (e.g., history of seizures, chronic headaches, pregnancy) ${ }^{26,27}$.

2. When the subject arrives, instruct the subject about the fMRI experiment details and describe the experience to expect (e.g., visual stimulus, tingling or phosphenes from tACS, special task instructions).

3. Place electrodes according to the 10-20 EEG system and stimulator preparation.

1. Using a tape measure, measure the distance on the subject's head from the nasion to the inion, and from ear-to-ear, over the top of the head. The intersection of both lengths gives the position on the head for $\mathrm{Cz}$, according to the 10-20 EEG system. Mark the spot for $\mathrm{Cz}$ on the scalp using a marker.

2. Place an EEG cap without electrodes on the subject's head, with $\mathrm{Cz}$ aligned to the mark on the subject's scalp, determine the desired location of the electrodes and mark them.

NOTE: It is important that all experimenters use the same placement system to ensure consistency through all experiments; the 10-20 EEG system, which is commonly used in transcranial stimulation experiments, has specific guidelines to maintain accurate electrode placement $^{26,28}$.

3. Using alcohol and cotton pads, clean the hair and skin on and around the marked spots on the subject's scalp; remove oils and hair products.

4. Spread some gel on the rubber electrodes and press each electrode firmly on the marked and cleaned locations on the subject's scalp, ensuring full contact from electrode to conductive gel to the scalp with minimal impedance.

5. Using a spare shielded LAN cable, connect the filter boxes and MR-safe cables to the stimulator and to the rubber electrodes as depicted in Figure 1A

6. Turn on the stimulator and test the impedance (refer to user's manual for details). If the impedance is not below $20 \mathrm{k} \Omega$, press the electrodes on the scalp or add electrode gel as necessary until this impedance guideline is met.

7. When the impedance is below $20 \mathrm{k} \Omega$, allow the stimulator to output current for a few seconds to familiarize the subject with the sensory experience. Ask the subject about sensory perception during this test, including whether tingling sensation exists and can be withstood, and extent or location of phosphenes during stimulation.

8. At this point, the subject is prepared to move to the scanner bed. Leaving the electrode cable plugged into the rubber electrodes on the subject, disconnect the stimulator, the spare LAN cable, and the outer and inner filter boxes. 
9. Connect the outer filter box to the LAN cable that runs through the waveguide to the MR scanner, leaving as little exposed LAN cable outside of the waveguide as possible (see Figure 1B). Connect the stimulator to the outer filter box using the stimulator cable and double-check that the stimulator is connected to the presentation computer trigger output.

4. Prepare the subject inside the MR scanner.

NOTE: Figure 1C shows the full tACS-fMRI setup during the experiment. It is critical to arrange the cables and inner filter box as specified, with the electrode cable arranged at an approximately $90^{\circ}$ angle to the plane of the scanner bed and the inner filter box resting on the scanner bed railing on the right side of the scanner bore. Neglecting to do so can damage the safety circuit of the electrode cable; this configuration applies for both open and closed RF coils.

1. After ensuring that the subject is free of magnetic materials and ready for the MRI experiment, lead the subject into the scanner room.

2. Give ear plugs for hearing protection to the subject, and instruct the subject to lie on the scanner bed, placing pillows around and under the head and under the legs for comfort and to reduce movement. When placing the pillows behind the subject's head, pay special attention to lay the electrode cable flat and in a position that is comfortable for the subject to lie on for the duration of the experiment.

3. Give the alarm ball and MR-safe response button box to the subject to hold such that minimal movement is required to push a button to respond in the experiment.

4. Secure the RF head coil over the subject's head with a mirror attached such that the subject can see the projection screen reflected in the correct orientation.

5. Temporarily secure the free end of the electrode cable coming from the rubber electrodes to a place in the head coil such that it does not catch when the bed is moving. Figure 1D shows the subject's head positioned in the head coil with pillows, mirror, and tACS cable in place before moving the bed to the center head coil for imaging. The filter box is also shown placed on the scanner bed railing, as an example of where it must sit relative to the head coil when the scanner bed is in measurement position.

6. Move the scanner bed into measurement position. From the back end of the scanner bore, connect the electrode cable from the rubber electrodes to the inner filter box that connects to the LAN cable, as depicted in Figure 1C. To prevent excess motion during scanning, secure the cables and filter box along the scanner bed railing to the right of the bore with tape and sand bags. Place projector screen into the rear end of the scanner bore.

7. Test the impedance on the stimulator once more to ensure that all connections between cables, filter boxes, and the stimulator are made properly.

\section{MR Scanning and Experiment}

1. Before the scan begins, test that the presentation computer registers when the subject pushes response buttons.

2. Acquire high-resolution T1-weighted anatomical data (e.g., three-dimensional turbo fast low angle shot, echo time (TE): 3.26 ms, repetition time (TR): $2,250 \mathrm{~ms}$, inversion time: $900 \mathrm{~ms}$, flip angle $9^{\circ}$, isotropic resolution of $1 \times 1 \times 1 \mathrm{~mm}^{3}$ ).

1. After the acquisition, adjust contrast and windowing on the anatomical MRI to low and high extremes to visually detect noise during scanning that may result from the stimulator setup. Continue this visual monitoring of noise concurrently with functional image acquisition.

3. Start the experiment on the presentation computer, ready to begin with the scanner trigger, and start the stimulator to wait for the presentation computer output trigger. Leave the stimulator on and connected throughout the fMRI experiment to avoid differences in the temporal signalto-noise ratio (tSNR) between stimulator on and off conditions ${ }^{22}$.

4. Start the fMRI scan (e.g., two-dimensional T2*-weighted gradient-echo echo-planar imaging; TE: $30 \mathrm{~ms}, \mathrm{TR}: 2,000 \mathrm{~ms}$, flip angle $70^{\circ}, 33$ slices of 3-mm thickness, no gap between slices at an in-plane resolution of $3 \times 3 \mathrm{~mm}^{2}, 210$ volumes for seven minutes of scanning), which triggers the start of the experiment on the presentation computer. Monitor the stimulator display to guarantee that current is sent at desired times throughout the experimental runs.

\section{Experiment Conclusion}

1. After the experiment has run and scanning is finished, unplug the inner filter box from the cable connected to the rubber electrodes before moving the scanner bed, remove the subject from the scanner, and remove the electrodes, leaving the subject free to wash their hair.

2. Turn off the stimulator, and plug it in to recharge. Clean the rubber electrodes with water for their next use. 


\section{Representative Results}

Figure 2 and Figure 3 show representative images acquired for equipment noise tests in a phantom and in a human subject, respectively. In every row, Figure 2 and Figure $\mathbf{3}$ show representative axial slices from an acquired volume or calculated map, labeled accordingly above the row. The rightmost image on each row is a sagittal representation of the corresponding volume or calculated map, indicating axial slice locations with blue lines. Aside from the first row, which illustrates electrode placement in white, the volume is overlaid on a T1-weighted image in each figure. Notice that there is no distortion or signal dropout from the electrodes in the T1-weighted images. The second row of Figure 2 shows representative functional MRI data acquired with the tACS setup in place and turned on. In the phantom in Figure 2, notice there is some signal dropout and distortion due to the electrodes, however, row 2 of Figure 3 shows that these distortions do not extend beyond the scalp in a subject. Rows three and four of Figure 2 show noise measurements in the volume, which are acquired using the same parameters as the fMRI data, but without an RF excitation pulse. The images show the noise level in the scanner room and of the MR hardware during the scan. Row three is a noise measurement with tACS off, and row four is one with tACS on. In the fifth and sixth row of Figure 2 are tSNR maps for functional runs with the tACS setup and the stimulator off and on, respectively. TSNR maps calculated from data acquired in the human subject appear in Figure 3 rows three, with tACS off, and four, with tACS on. Notice there is no visible difference in intensity when comparing between stimulation conditions. As we demonstrated in a previous study, the tACS equipment produces around $5 \%$ drop in tSNR in images compared to those acquired without the tACS setup, however the tSNR should remain stable across stimulation on and off conditions ${ }^{22}$.

Figure 4 represents a series of images that demonstrates signal dropout that can occur when non-MR-compatible electrodes are used. Slices from an fMRI volume acquired of a subject with electrodes that may have some metal contaminations show signal dropout below the electrode placed roughly over primary motor cortex, as indicated with red circles.

Figure 5 shows results of an experiment testing the effects of current strength of $16 \mathrm{~Hz} \mathrm{Cz-Oz} \mathrm{tACS}$ on the BOLD signal in subjects whose only task is central cross fixation. Throughout the experiment, 12-second periods of tACS were interleaved with non-stimulation periods varying from 24 - 32 seconds. In a pseudorandomized order, tACS was applied with a different current strength $(500 \mu \mathrm{A}, 750 \mu \mathrm{A}, 1,000 \mu \mathrm{A}, 1,500 \mu \mathrm{A})$ in each of four runs. Figure 5A shows event-related averages of the BOLD signal for statistically significant clusters, with increasing effect on the BOLD signal with increased current strength. Additionally, Figure 5B shows current-strength specific T-score maps illustrating regional specificity of effects as well as increasing spatial effect with increased current strength. It is also worthwhile to note that BOLD activity in frontal regions was changed significantly, showing that modulations were not always directly below the electrodes. For details, refer to Cabral-Calderin and colleagues $^{22}$.

Figure 6 shows representative results of an experiment testing the frequency dependence of tACS effects during a visual perception task. Subjects reported the perceived direction of a bistable rotating sphere. At the same time, tACS was applied with electrodes placed at $\mathrm{Cz}$ and $\mathrm{Oz}$ at one of three stimulation frequencies $(10 \mathrm{~Hz}, 60 \mathrm{~Hz}$, or $80 \mathrm{~Hz})$ in each of three separate sessions. Figure $6 \mathrm{~A}$ illustrates the experiment timing with visual presentation and tACS periods between blocks of central cross fixation. TACS condition and frequency effect interaction maps and cluster post-hoc tests show frequency-specific effects in the parietal cortex, with $10 \mathrm{~Hz}$ tACS decreasing and $60 \mathrm{~Hz}$ increasing signal (Figure 6B). Figure 6C shows T-score maps of specific effects of $60 \mathrm{~Hz}$ tACS extending beyond the parietal cortex to include some occipital and frontal regions. For experiment and analysis details, refer to Cabral-Calderin, et al. ${ }^{22}$. 

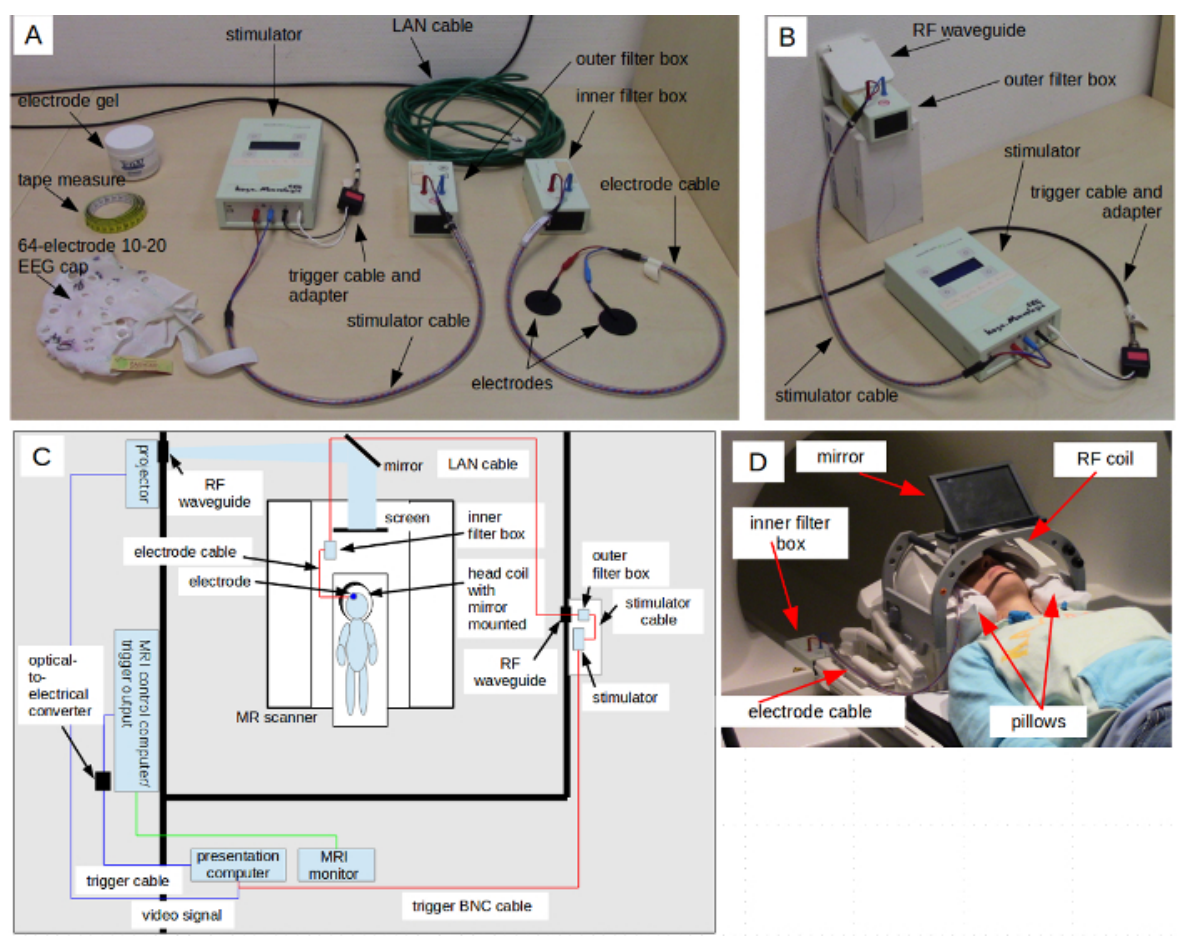

Figure 1: TACS Setup in the Scanner. (A) TACS Setup with all Necessary Elements. The stimulator and cables are connected outside of the MR shielded room. Also shown are the EEG cap, tape measure, and conductive gel used for electrode placement. (B) Outer Filter Box and Stimulator Placed Outside the Scanner Room. The LAN cable (not visible in the figure) comes from the scanner room through the RF waveguide tube and connects to the outer filter box, with as little LAN cable exposed outside of the scanner room as possible. The stimulator should be connected to the outer filter box as well as to the presentation computer trigger output cable. (C) Scanner Environment with Experimental Setup. Depiction of tACS setup, including presentation computer, scanner computer and trigger output, and projector. (D) Subject Positioning for Experiment. Important elements include pillows, cable placement, viewing mirror, and head coil. Filter box is placed on scanner bed railing as an example of placement inside the bore. Please click here to view a larger version of this figure. 


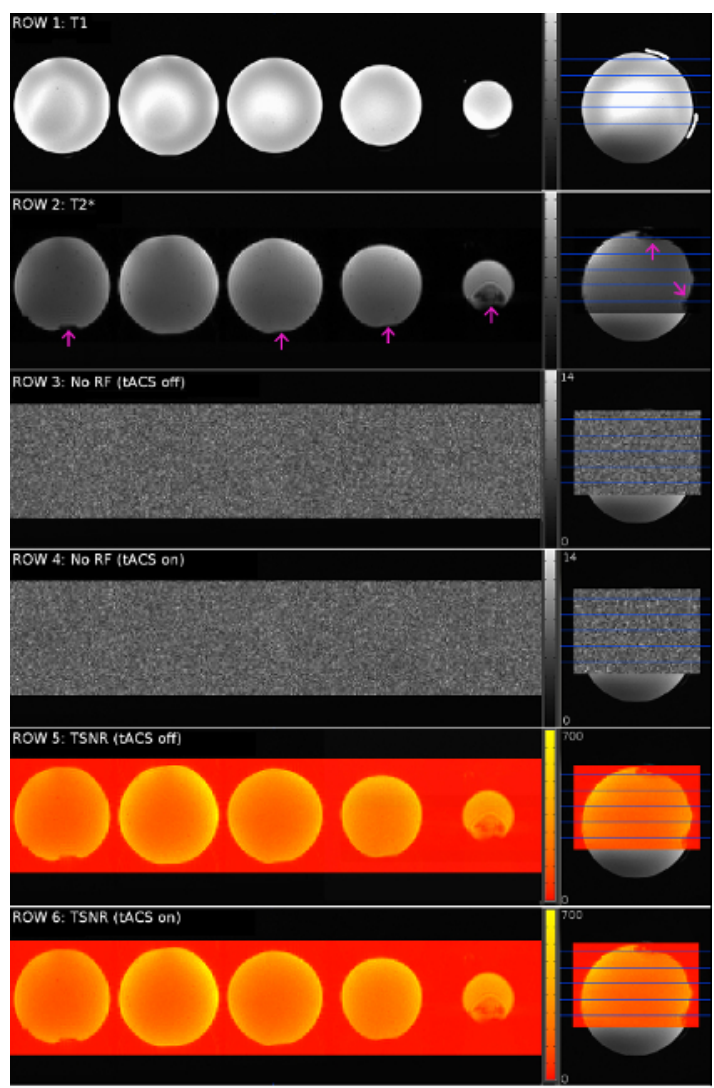

Figure 2: Quality Assessment MR Images Acquired of a Phantom. Row 1: High-resolution anatomical T1-weighted image axial slices with their positions indicated by blue lines on a sagittal slice to the right (also seen in each following row). On the sagittal plane, electrode positions are illustrated in white. Row 2: T2*-weighted echo-planar image slices, with magenta arrows indicating signal dropout and distortion due to electrodes and/or electrode gel. On the sagittal plane, the positioning of the corresponding volume is shown as an overlay (also seen in each following row). Row 3: Noise image slices acquired with fMRI experimental parameters and no RF excitation pulse while the tACS setup is in place and turned on, but not stimulating. Row 4: No-RF-excitation image acquired with tACS setup in place and stimulator on and stimulating at $16 \mathrm{~Hz}$. Row 5: TSNR map calculated from data acquired with the tACS setup in place and turned on, but not stimulating. Row 6: TSNR map calculated from data acquired with the tACS setup in place and stimulating at $16 \mathrm{~Hz}$. Please click here to view a larger version of this figure.

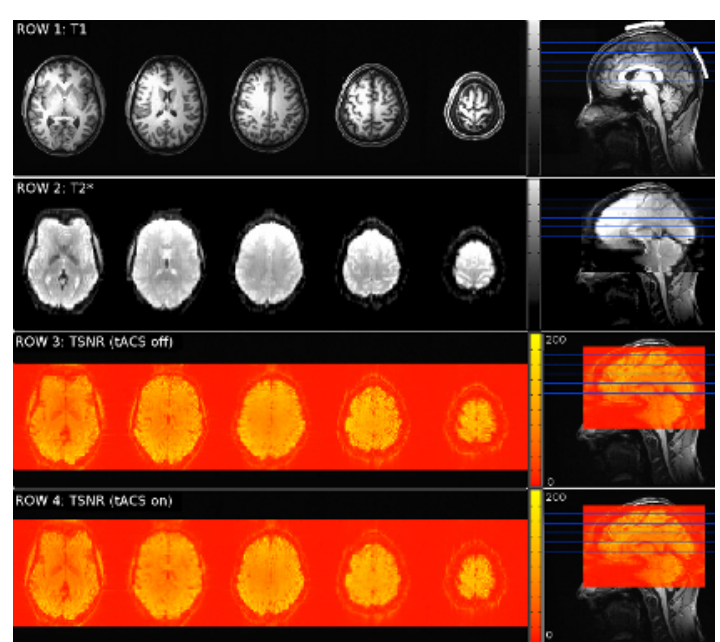

Figure 3: Quality Assessment MR Images Acquired of a Subject. Row 1: High-resolution anatomical image axial slices with their positions indicated by blue lines on a sagittal slice to the right (as seen in each row). Electrode positions are illustrated in white on the sagittal view. Row 2 : T2*-weighted echo-planar image slices showing no signal dropout due to electrodes and/ or electrode gel. On the sagittal plane, the positioning of the corresponding volume is shown as an overlay (also seen in each following row). Row 3: TSNR map calculated from data acquired with the tACS setup in place and turned on, but not stimulating. Row 4: TSNR map calculated from data acquired with the tACS setup in place and stimulating at $16 \mathrm{~Hz}$. Please click here to view a larger version of this figure. 


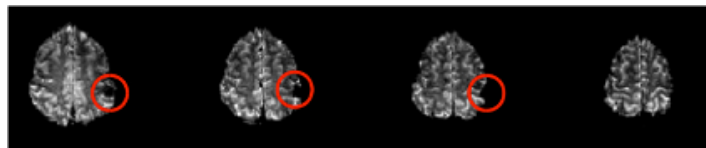

Figure 4: Signal Dropout Due to a Contaminated Electrode. Slices from an fMRI volume acquired of a subject using a contaminated electrode placed roughly over the hand knob of the motor cortex. Red circles indicate regions below the electrode with signal dropout. Please click here to view a larger version of this figure.

A

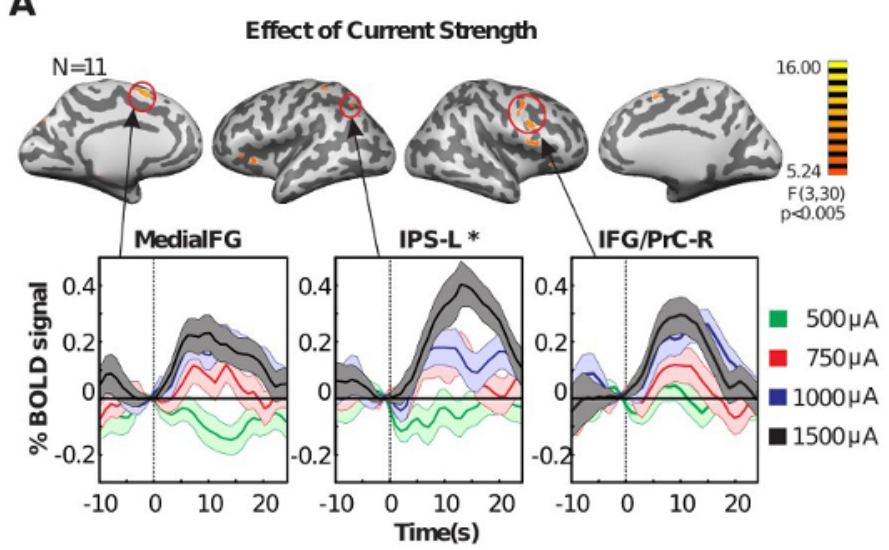

B

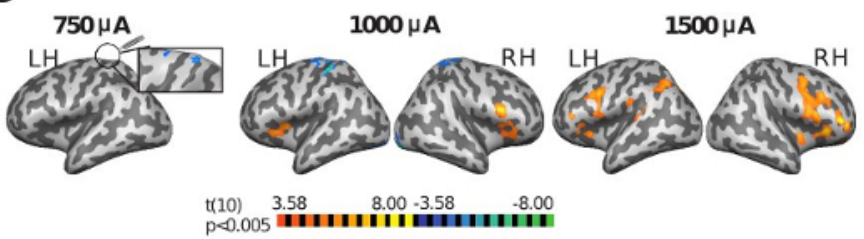

Figure 5: Effect of Current Strength on tACS Modulation of the BOLD Signal. (A) F-score Maps Showing the Main Effect of Current Strength on the Effect of $16 \mathrm{~Hz}$ tACS. A significant main effect of current strength in a one-way rANOVA [within factor: current strength (500, 750, $1,000,1,500 \mu \mathrm{A})]$ is apparent. The plots show the event-related average time course of the BOLD signal for the tACS-on periods for each current strength. Shaded regions indicate standard error of the mean across subjects. MedialFG = medial frontal gyrus, IPS = intraparietal sulcus, IFG $=$ inferior frontal gyrus, $\mathrm{PrC}=$ precentral gyrus, $\mathrm{L}=$ left, $\mathrm{R}=$ right, ${ }^{*}$ cluster not corrected for multiple comparisons. (B) T-score Maps Showing BOLD Activity Changes during $16 \mathrm{~Hz}$ tACS for Each Current Strength. No significant effect was found with $500 \mu \mathrm{A}$ tACS. LH = left hemisphere; RH = right hemisphere. This picture has been modified from Cabral-Calderin et al. ${ }^{29}$. Please click here to view a larger version of this figure. 

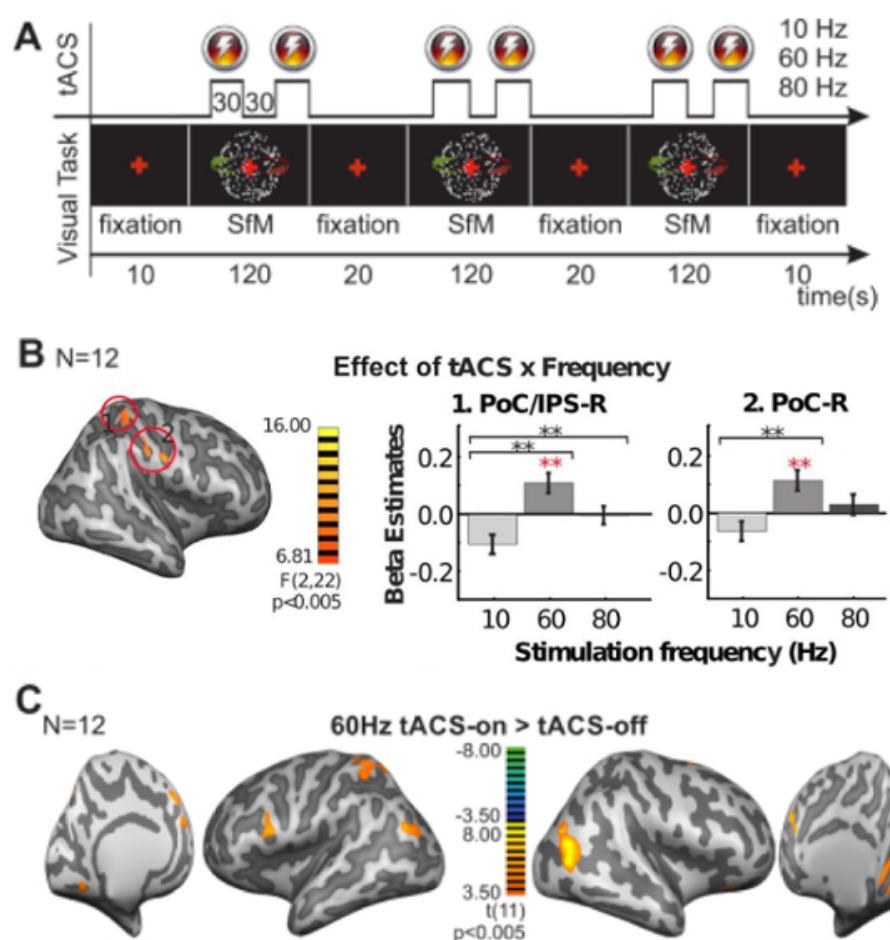

Stimulation frequency $(\mathrm{Hz})$

Figure 6: Effect of tACS on the BOLD Signal in a Visual Perception Task. (A) Schematic Representation of the Experiment. Visual stimulus and tACS were applied in a block design, with $30 \mathrm{~s}$ on-off tACS blocks occurring during $120 \mathrm{sec}$ blocks of visual stimulus presentation. Each frequency was tested in a different session. SfM = structure-from-motion. (B) TACS Condition and Frequency Interaction Effect. F-statistic maps showing significance in two-way rANOVA [within factors: tACS (on, off), frequency $(10 \mathrm{~Hz}, 60 \mathrm{~Hz}, 80 \mathrm{~Hz}$ )] and beta estimates for two representative clusters in the post-central gyrus. Continuous lines and black asterisks mark significant differences for post-hoc comparisons for tACS on-off interaction effects of $10 \mathrm{~Hz}$ versus $60 \mathrm{~Hz}$ and $10 \mathrm{~Hz}$ versus $80 \mathrm{~Hz}$, and red asterisks imply a significant difference for tACS on versus off post-hoc tests. PoC = postcentral gyrus, IPS = intraparietal sulcus. (C)T-score Map of $60 \mathrm{~Hz}$ tACS. Significant differences comparing $60 \mathrm{~Hz}$ tACS on versus off. This picture has been reprinted from Cabral-Calderin et al. ${ }^{29}$. Please click here to view a larger version of this figure.

\section{Discussion}

Here, we have described the procedure for a simultaneous tACS-fMRI experiment setup and execution using an MR-compatible tACS system. Some steps in this procedure require special attention, especially with respect to the subject setup. The MR-compatible stimulator and setup used in this experiment has a minimum impedance of approximately $12 \mathrm{k} \Omega$ with the cables, filter boxes, and electrodes only, and the manufacturer recommends $20 \mathrm{k} \Omega$ minimum impedance with electrodes connected to the subject; this requirement depends on stimulator product and manufacturer. When applying electrodes to the subject, if the impedance is too high, some steps can be taken to reduce this value aside from pressing the electrodes. For example, it may be easier to first cover the marked and cleaned locations on the scalp with electrode gel, including the hair, before pressing the electrode onto the scalp. This will ensure current spread across the non-conductive material; however, be careful to limit electrode gel coverage to approximately the same surface area as the electrodes to direct current spread to the desired region of stimulation. Pay particular attention to this if the electrodes are close together, because current shunting between the electrodes may occur through excess electrode gel contact. If the electrode is on the back of the head where the subject will be laying directly on it, special care must be taken to place pillows behind the head such that the subject will not grow uncomfortable as the experiment continues; this discomfort may not be a problem initially for the subject, however experience shows that pain arises and increases with time. Additionally, as with all fMRI experiments, subject motion introduces problematic confounds, so it is important that the subject is comfortable with all the cables and electrodes in place.

The most important aspect of the setup to consider is the noise potentially introduced into the MR environment that can induce image artifacts and distortions. Prior to the experiment, it is prudent to test for image artifacts with the whole tACS setup in place. A normal spherical phantom can be used, securing electrodes with electrode gel. It is important to provide some way for the current to travel between electrodes, which can be accomplished by applying a generous amount of electrode gel in a path from one electrode to the other. Run the entire experiment, as planned for the subject, including parameter variations such as frequency and current. During the scanning session, adjusting contrast and windowing to extremes in the image viewer on the MR scanner control computer allows easier visual detection of noise. When visually monitoring for noise before and during the experiment, noise may occur as spikes in the image with high intensity, patterns where signal should not be measured, or varying intensity over time, as examples. Acquiring fMRI data with the RF excitation pulse turned off gives information about scanner environment noise during scanning without acquiring the actual image signal (see Figure 2). This noise test can be done in every scanning session. If there are variations in the noise, check that all cables are intact and well connected to the stimulator, electrodes, and filter boxes. No cables should sit in loops. Noise or distortion can arise from broken cables, electrodes with metal contaminants in the rubber (despite being sold as MR-compatible), and faulty connections, among other possibilities. The stimulator is battery-driven to minimize electrical noise in the setup; ensure that it is fully charged before every experiment and that it stays on and connected throughout the experiment. TSNR in functional images will decrease around $5 \%$ with the stimulator connected, however, values should be stable across stimulation conditions ${ }^{22}$. Simultaneous transcranial electrical stimulation-fMRI tests on cadavers have shown that there are no artifacts associated with alternating current 
stimulation, which is an advantage compared to direct current stimulation ${ }^{30}$. Theoretically, this lack of artifacts can be explained by a net current of zero at the time the image is acquired ${ }^{30}$. However, for some of the experiments conducted in our lab, the acquisition time or TR is not a multiple of the stimulation frequency. After conducting the noise tests mentioned in this protocol and examining images for artifacts, which were not visible, we concluded that any difference in net current from zero is small and too negligible to induce artifacts.

Another critical point for successful experiments is that the presentation computer receives the trigger output of the scanner and that the stimulator receives the trigger from the presentation computer. Prior to the experiment, program the visual stimulus design and timing using the desired software. This program must use triggers to synchronize the visual stimulus presentation with the MR scanner and the stimulator; it initiates with a trigger that is output from the MR scanner and also sends output triggers to the stimulator at desired stimulation times. An easy way to check trigger communication during setup is to use an oscilloscope attached with a BNC cable to the scanner trigger output as well as the presentation computer output. In our setup, the MR scanner outputs a trigger (toggle) for every functional volume acquired, and the presentation computer outputs a signal as programmed through the presentation software. The analysis of a well-designed experiment rests critically on properly timed stimulation.

Some steps of this experiment may be adapted as necessary for the laboratory setting requirements. For example, this setup describes using a projector and mirrors for visual stimulus presentation, however the visual stimulus output device can be MR-safe liquid-crystal-display goggles or an MR-safe monitor, chosen based on experiment and lab preferences or limitations. Also, MRI scan parameters should be tailored to the experiment. It is worthwhile to note that attention should be given to the appropriate choice of experimental control for tACS, although a straightforward answer does not exist. A short sham stimulation of 30 seconds can mimic the somatosensation induced by tACS that diminishes eventually with prolonged stimulation; however, some studies show that even short periods of stimulation can induce oscillatory entrainment ${ }^{12}$. Another possible control that can be used for tACS is to stimulate using a non-effective frequency, or, in other words, a different frequency from the one of interest. The exception here would be that somatosensation and phosphene perception vary according to stimulation frequency ${ }^{31}$. Finally, regarding subjective experiences of stimulation, tACS-induced phosphenes vary across individuals, so in order to best capture subject variability, consider using a detailed rating system for phosphene perception, and spend some time with the subject describing the various features of phosphenes (e.g., location, intensity) that can arise so that the subject can attentively evaluate his or her experience during stimulation $^{32,33}$

The representative results shown here suggest that tACS effects are current dependent, frequency dependent, and that modulation is not limited to the regions below the electrodes, but extends to distant, likely functionally connected regions. One limitation of this technique is the temporal resolution of $\mathrm{fMRI}$ as well as of the BOLD response. The data acquisition and the hemodynamic response are not as fast as the stimulation frequency or electrical activity of the brain, so direct interactions with frequency-specific effects of tACS cannot be measured. However, given that the greatest share of scientific literature of tACS effects is of behavioral studies, and that tACS obviously affects a whole, complicated neural system, it is clear that simultaneous tACS-fMRI experiments have much to offer for informing us about tACS effects in the brain. EEG and MEG offer insights on the level of temporal resolutions that match those of neural activity. However, EEG and MEG suffer from spatial resolution and cortical depth limitations or computationally intensive source-reconstruction techniques. Stimulation frequency and harmonic artifacts overriding brain signals of interest recorded at the same frequencies further complicate EEG and MEG analyses. Innovative workarounds have been applied to tackle some of these challenges. Helfrich et al. employed a novel technique to remove the tACS artifact from EEG data using an artifact template subtraction and principle component analysis ${ }^{15}$. They showed that $10 \mathrm{~Hz}$ tACS applied parieto-occipitally increases alpha activity in the parietal and occipital cortices and induces synchrony in cortical oscillators functioning at similar intrinsic frequencies. Witkowski and colleagues applied amplitude-modulated tACS and successfully created MEG-based cortical maps of entrained brain oscillations ${ }^{34}$. With the goal of applying tACS in research for better understanding normal and abnormal brain function, and eventually clinically for diagnostics or therapeutics, tACS should be separately combined with EEG, MEG, and fMRI to complementarily establish best practices for specific desired effects that can be tailored specifically to individuals. When such practices are established, effective investigations can be carried out to better understand the function of neural oscillations (e.g., clearly defining functional roles and relationships of different frequency bands) and their modulation with tACS (e.g., whether the mechanism occurs through entrainment or plastic changes ${ }^{35}$ ).

Considering future directions, the setup described here is tailored for fMRI experiments studying perception or cognition, as the structurefrom-motion study described here and others have demonstrated. Cabral-Calderin and colleagues showed that activation in regions of the occipital cortex was dependent upon task and tACS frequency in a video-watching versus finger-tapping experiment ${ }^{22}$. In a simultaneous tACSresting-state fMRI study, Cabral-Calderin and colleagues showed frequency-dependent effects of tACS on intrinsic functional connectivity and resting state networks ${ }^{23}$. Vosskuhl et al. combined tACS and fMRI to show BOLD decrease during a visual vigilance task at individual alpha frequency stimulation ${ }^{24}$. Alekseichuk and colleagues showed that immediate aftereffects of $10 \mathrm{~Hz}$ tACS modulate the BOLD signal during a visual perception of checkered rings and wedges, indicating a change in the neural metabolism of a passive perception task ${ }^{36}$. These studies set the stage for simultaneous tACS-fMRI studies to probe functional mechanisms on many levels, from metabolism to cognition. At such an early stage in the use of tACS for translational research, there is much potential for simultaneous tACS-fMRI experiments to add to the understanding of both the stimulation technique and the contribution of oscillations to cognitive functions.

\section{Disclosures}

The authors have nothing to disclose.

\section{Acknowledgements}

We thank Ilona Pfahlert and Britta Perl for technical assistance during functional imaging experiments and Severin Heumüller for excellent computer support. This work was supported by the Herman and Lilly Schilling Foundation and the Center for Nanoscale Microscopy and Molecular Physiology of the Brain (CNMPB). 


\section{References}

1. Thut, G. Modulating brain oscillations to drive brain function. PLoS Biol. 12 (12), e1002032 (2014).

2. Joundi, R. A., Jenkinson, N., Brittain, J. S., Aziz, T. Z., \& Brown, P. Driving Oscillatory Activity in the Human Cortex Enhances Motor Performance. Current Biology. 22 (5), 403-407 (2012).

3. Jausovec, N., \& Jausovec, K. Increasing working memory capacity with theta transcranial alternating current stimulation (tACS). Biological Psychology. 96 42-47 (2014).

4. Feurra, M., Paulus, W., Walsh, V., \& Kanai, R. Frequency specific modulation of human somatosensory cortex. Frontiers in Psychology. 2 (2011).

5. Laczo, B., Antal, A., Niebergall, R., Treue, S., \& Paulus, W. Transcranial alternating stimulation in a high gamma frequency range applied over V1 improves contrast perception but does not modulate spatial attention. Brain stimulation. 5 (4), $484-491$ (2012).

6. Cabral-Calderin, Y., Schmidt-Samoa, C., \& Wilke, M. Rhythmic Gamma Stimulation Affects Bistable Perception. Journal of Cognitive Neurosci. 27 (7), 1298-1307 (2015).

7. Kanai, R., Chaieb, L., Antal, A., Walsh, V., \& Paulus, W. Frequency-dependent electrical stimulation of the visual cortex. Curr Biol. 18 (23), 1839-1843 (2008)

8. Brittain, J. S., Probert-Smith, P., Aziz, T. Z., \& Brown, P. Tremor Suppression by Rhythmic Transcranial Current Stimulation. Current Biology. 23 (5), 436-440 (2013).

9. Sabel, B. A. et al. Non-invasive alternating current stimulation improves vision in optic neuropathy. Restorative Neurology Neuroscience. 29 (6), 493-505 (2011).

10. Fedorov, A., Chibisova, Y., Gall, C., \& Sabel, B. A. Non-Invasive Alternating Current Stimulation Induces Recovery From Stroke. Brain Injury. 26 (4-5), 634 (2012)

11. Reato, D., Rahman, A., Bikson, M., \& Parra, L. C. Low-Intensity Electrical Stimulation Affects Network Dynamics by Modulating Population Rate and Spike Timing. Journal of Neuroscience. 30 (45), 15067-15079 (2010).

12. Reato, D., Rahman, A., Bikson, M., \& Parra, L. C. Effects of weak transcranial alternating current stimulation on brain activity-a review of known mechanisms from animal studies. Front Hum Neurosci. 7687 (2013).

13. Herrmann, C. S., Rach, S., Neuling, T., \& Struber, D. Transcranial alternating current stimulation: a review of the underlying mechanisms and modulation of cognitive processes. Front Hum Neurosci. 7279 (2013).

14. Alagapan, S. et al. Modulation of Cortical Oscillations by Low-Frequency Direct Cortical Stimulation Is State-Dependent. PLoS Biol. 14 (3), e1002424 (2016).

15. Helfrich, R. F. et al. Entrainment of brain oscillations by transcranial alternating current stimulation. Curr Biol. 24 (3), $333-339$ (2014).

16. Neuling, T., Zaehle, T., \& Herrmann, C. Simultaneous recording of EEG and transcranial electric stimulation. International Journal of Psychophysiology. 77 (3), 312 (2010).

17. Zaehle, T., Rach, S., \& Herrmann, C. S. Transcranial alternating current stimulation enhances individual alpha activity in human EEG. PLoS One. 5 (11), e13766 (2010).

18. Neuling, T. et al. Friends, not foes: Magnetoencephalography as a tool to uncover brain dynamics during transcranial alternating current stimulation. Neuroimage. 118 406-413 (2015).

19. Ruhnau, P., Keitel, C., Lithari, C., Weisz, N., \& Neuling, T. Flicker-Driven Responses in Visual Cortex Change during Matched-Frequency Transcranial Alternating Current Stimulation. Front Hum Neurosci. 10184 (2016).

20. Witkowski, M. et al. Mapping entrained brain oscillations during transcranial alternating current stimulation (tACS). Neuroimage. 140 89-98 (2016).

21. Alekseichuk, I., Diers, K., Paulus, W., \& Antal, A. Transcranial electrical stimulation of the occipital cortex during visual perception modifies the magnitude of BOLD activity: A combined tES-fMRI approach. Neuroimage. 140 110-117 (2016).

22. Cabral-Calderin, Y. et al. Transcranial alternating current stimulation affects the BOLD signal in a frequency and task-dependent manner Hum Brain Mapp. 37 (1), 94-121 (2016).

23. Cabral-Calderin, Y., Williams, K. A., Opitz, A., Dechent, P., \& Wilke, M. Transcranial alternating current stimulation modulates spontaneous low frequency fluctuations as measured with fMRI. Neuroimage. 141 88-107 (2016).

24. Vosskuhl, J., Huster, R. J., \& Herrmann, C. S. BOLD signal effects of transcranial alternating current stimulation (tACS) in the alpha range: A concurrent tACS-fMRI study. Neuroimage. 140 118-125 (2016).

25. Krause, B., \& Cohen Kadosh, R. Not all brains are created equal: the relevance of individual differences in responsiveness to transcranial electrical stimulation. Frontiers in Systems Neuroscience. 8 (25) (2014).

26. DaSilva, A. F., Volz, M. S., Bikson, M., \& Fregni, F. Electrode positioning and montage in transcranial direct current stimulation. $J$ Vis Exp. (51) (2011).

27. Nitsche, M. A. et al. Safety criteria for transcranial direct current stimulation (tDCS) in humans. Clinical Neurophysiology. 114 (11), $2220-2222$ (2003).

28. Poeppl, T. B. et al. Connectivity and functional profiling of abnormal brain structures in pedophilia. Hum Brain Mapp. 36 (6), $2374-2386$ (2015).

29. Cabral-Calderin, Y. et al. Transcranial Alternating Current Stimulation Affects the BOLD Signal in a Frequency and Task-dependent Manner. Human Brain Mapping. 37 (1), 94-121 (2016).

30. Antal, A. et al. Imaging artifacts induced by electrical stimulation during conventional fMRI of the brain. Neuroimage. 85 Pt $31040-1047$ (2014).

31. Turi, Z. et al. Both the cutaneous sensation and phosphene perception are modulated in a frequency-specific manner during transcranial alternating current stimulation. Restor Neurol Neurosci. 31 (3), 275-285 (2013).

32. Kanai, R., Paulus, W., \& Walsh, V. Transcranial alternating current stimulation (tACS) modulates cortical excitability as assessed by TMSinduced phosphene thresholds. Clinical Neurophysiology. 121 (9), 1551-1554 (2010).

33. Schutter, D. J., \& Hortensius, R. Retinal origin of phosphenes to transcranial alternating current stimulation. Clinical Neurophysiology. 121 (7), 1080-1084 (2010).

34. Witkowski, M. et al. Mapping entrained brain oscillations during transcranial alternating current stimulation (tACS). Neuroimage. (2015). 
35. Vossen, A., Gross, J., \& Thut, G. Alpha Power Increase After Transcranial Alternating Current Stimulation at Alpha Frequency (alpha-tACS) Reflects Plastic Changes Rather Than Entrainment. Brain Stimul. 8 (3), 499-508 (2015).

36. Alekseichuk, I., Diers, K., Paulus, W., \& Antal, A. Transcranial electrical stimulation of the occipital cortex during visual perception modifies the magnitude of BOLD activity: A combined tES-fMRI approach. Neuroimage. (2015). 